\title{
Modificaciones producidas en las proteínas alimentarias por su interacción con lípidos peroxidados. III. Consecuencias nutricionales y toxicológicas
}

\author{
Por F.J. Hidalgo, R. Zamora y J. Girón \\ Instituto de la Grasa y sus Derivados. C.S.I.C. \\ Avda. Padre Garcia Tejero, 4 \\ 41012-Sevilla
}

RESUMEN

Modificaciones producidas en las proteinas alimentarias por su interacción con lípidos peroxidados. III. Consecuencias nutricionales y toxicológicas.

Las consecuencias nutricionales y toxicológicas de las interacciones lípido (oxidado)-proteína son examinadas en esta parte. La influencia de estas reacciones en la calidad de las proteinas y en la asimilabilidad y tránsito metabólico de varios animoácidos en ensayos con animales de experimentación son utilizados para discutir la importancia real de estas reacciones.

PALABRAS CLAVE: Aminoácido (pérdida) - Consecuencias toxicologicas - Efecto nutricional - Información (articulo) - Oxidación de metionina.

\section{SUMMARY}

Modifications produced in food proteins following interactions with oxidizing lipids. III. Nutritional and toxicological consequences.

The nutritional and toxicological consequences of oxidizing lipid-protein interactions are reviewed in this part. Influence on protein quality and on the availability and metabolic transit of several amino acids as measured in animal assays are used to discuss the real importance of these reactions.

KEY-WORDS: Amino acid (loss) - Information (paper) - Methionine oxidation - Nutritional effect - Toxicological consequences.

\section{INTRODUCCION}

Las partes I y II de esta serie describieron cómo la oxidación lipídica se produce en alimentos que contienen ácidos grasos poliinsaturados y cómo estos productos de oxidación lipídica reaccionan con las proteínas (1) (2).

La oxidación lipídica se puede considerar que ocurre en diversas etapas que son consecutivas al mismo tiempo que simultáneas. La primera consiste en la formación de unos productos primarios de oxidación, principalmente hidroperóxidos. Esta etapa es seguida por la degradación radicalaria de los mismos para dar lugar a una serie de productos secundarios tales como aldehídos, hidrocarburos, y compuestos bi- y trifuncionales que pueden posteriormente evolucionar a su vez para dar compuestos más estables (1).
Tanto los lípidos peroxidantes como sus productos secundarios pueden reaccionar con proteínas (2), siendo los residuos de lisina, cist(e)ina, metionina, triptófano, tirosina e histidina los más afectados por estas reacciones en las proteínas. Así pues, las reacciones de interacción proteína-lípido oxidado producen, por tanto, una modificación de las proteínas alimenticias con consecuencias de pérdidas de su valor nutricional y de la formación de compuestos que pueden ser potencialmente tóxicos. El desarrollo de estos puntos será el tema de esta tercera parte de la serie.

\section{PERDIDA DE VALOR NUTRITIVO}

Las proteínas pierden valor nutritivo generalmente a causa de las reacciones de los aminoácidos lisina, metionina, cist(e)ina y triptófano. Estos aminoácidos que son esenciales nutricionalmente, o semiesenciales en el caso de la cisteína, y que, en muchas dietas, son limitantes, son los que interaccionan más fácilmente (3).

\subsection{Oxidación de la cist(e)ina}

Usando resonancia magnética nuclear, Finley y Ludin (4) (5) estudiaron la reacción de oxidación del residuo de cisteína en el glutatión producida por el hidroperóxido del ácido linoleico. Ellos detectaron cuatro productos de oxidación: cistina monoóxido y cistina dióxido, que fueron los mayoritarios, y pequeñas cantidades de ácido cisteínsulfínico y de ácido cisteico. Nada se conoce de la asimilación de cisteína monoóxido como fuente de cisteína en ratas (3). La cisteína dióxido es sólo $20-50 \%$ asimilable (6), mientras que el ácido cisteinsulfínico (7) y el ácido cisteico (8) no son asimilables.

Estos resultados están de acuerdo con los obtenidos por Nielsen et al. cuando estudiaron el valor biológico y la digestibilidad de nitrógeno en ratas usando una muestra de proteínas del suero bovino almacenadas con linoleato de metilo (9) (10). La asimilación de la cisteína se redujo un $28 \%$, indicando que la cisteína parece ser muy sensible a pérdidas de asimilabilidad como resultado de estas re- 
acciones de oxidación lipídica. Además estas pérdidas se producen en los primeros estadios de la oxidación lipídica (2). Por tanto, alimentos sometidos a ligera rancidez pueden presentar importantes deficiencias en cisteína (10).

\subsection{Oxidación de la metionina}

Por su interacción con lípidos peroxidantes los residuos de metionina son oxidados a metionina sulfóxido (2). Usando metionina sulfóxido marcada radiactivamente, Finot (11) encontró que no había diferencias significativas en los niveles de excreción e incorporación en los tejidos de la radioactividad cuando se comparaba con metionina sin oxidar, análogamente a los efectos nutricionales observados por Bennett (7) (12) (13), Miller et al. (14) y Gjoen y Njaa (15). Esto es probablemente debido a una reducción de metionina sulfóxido a metionina que ocurre antes de que sea utilizada en síntesis de proteínas. La localización de las enzimas responsables de esta reducción en hígado y riñón ha sido estudiada por Aymard et al. (16). Esta actividad enzimática del hígado está localizada principalmente en el citosol mientras que la actividad enzimática del riñón parece que está principalmente asociada a membranas y núcleos. En ambos casos el enzima es termolábil y su actividad aumenta por adición de NADH. Otros tejidos posiblemente también están implicados en los procesos de reducción de la metionina sulfóxido. Los estudios metabólicos llevados a cabo con animales vivos sugieren que bien la metionina sulfóxido es transportada a través de la pared intestinal y reducida durante su paso, o bien es reducida en el lumen y transportada como metionina libre.

Gjoen y Njaa (15) y Cuq et al. (17) han realizado estudios de crecimiento en ratas usando proteínas oxidadas. La ganancia de peso de ratas alimentadas con proteínas oxidadas de pescado resultó ser un $85 \%$ del valor obtenido con proteínas sin oxidar (15). La utilización proteica neta (UPN) y el coeficiente de eficacia proteica (CEP) de caseina oxidada (donde la metionina fue oxidada completamente a metionina sulfóxido) fueron un 10-15\% menores que para la caseina sin oxidar (17). Por su parte Nielsen et al. (9) (10), estudiando el valor biológico y la digestibilidad de nitrógeno en ratas usando proteínas de suero bovino almacenada con linoleato de metilo, encontraron que la asimilación de la metionina era sólo un $8 \%$ inferior a la asimilación de la proteína sin interaccionar. Todos estos resultados sugieren que la metionina sulfóxido enlazada a proteínas es sólo ligeramente menos asimilable biológicamente que la metionina sin oxidar y que se comporta en el proceso digestivo como metionina a pesar de su diferencia en estructura química.

Sin embargo, y a pesar de la análoga asimilabilidad y tránsito metabólico de residuos de metionina y metionina sulfóxido, la oxidación de este aminoácido es capaz de inducir cambios biológicos. Así, por ejemplo, en ratas alimentadas con dietas conteniendo metionina sulfóxido se ha observado importantes diferencias en el contenido de metionina libre en plasma (14) (15) y músculo (17). Asimismo, la presencia de metionina sulfóxido en los tejidos puede modificar la actividad de algunos enzimas tales como glutamina sintetasa (18) y $\gamma$-glutamilcisteína sintetasa (19) que son inactivadas in vitro por esta molécula.

\subsection{Oxidación del triptófano}

A diferencia de la lisina o metionina, la estabilidad de los resíduos de triptófano ha sido muy poco investigada, principalmente porque no existen métodos simples y porque la lisina y los aminoácidos azufrados son frecuentemente los aminoácidos más limitantes (20). La estabilidad de los residuos de triptófano a lípidos peroxidantes ha sido estudiado por O'Brien (21) y Kanazawa et al. (22) encontrando grandes pérdidas de triptófano. Más recientemente Nielsen et al. (23) (24) encontraron que la pérdida máxima de contenido de tritófano determinado químicamente en muestras de proteínas de suero bovino incubadas con linolenato de metilo peroxidante era de un $26 \%$. Las reducciones en triptófano asimilable fueron mucho mayores (hasta del $71 \%$ ) pero parecieron ser principalmente debidas a una caída global en la digestibilidad proteínica. El triptófano determinado químicamente multiplicado por la digestibilidad de nitrógeno estuvo bastante de acuerdo con los valores obtenidos de los ensayos con ratas. En otros experimentos con una cantidad de oxígeno limitada a $1 \mathrm{~mol}$ por mol lípido, la pérdida de triptófano se redujo grandemente hasta un $11 \%$. Esto sugiere que en alimentos almacenados donde la cantidad de oxígeno disponible es menor, las pérdidas de triptófano deben ser presumiblemente insignificantes.

\subsection{Pérdidas de lisina}

La lisina es un aminoácido muy sensible a la oxidación lipídica (25) (26) cuya pérdida se produce principalmente por reacción con los productos secundarios de la oxidación lipídica (2). Estudios de crecimiento y balance de nitrógeno en ratas han confirmado los resultados obtenidos químicamente cuando se estudiaron muestras de suero bovino incubadas con linolenato de metilo peroxidado (10). En estos casos un incremento de la actividad de agua y temperatura produjo una disminución del CEP y de la digestibilidad verdadera y biológica. Las pérdidas en asimilabilidad biológica fueron las más altas para la lisina, entre los aminoácidos estudiados, que llegó a disminuir a un $77 \%$ cuando la cantidad de oxígeno se redujo a $1 \mathrm{~mol}$ por mol de lípido.

Estudiando alimentos sujetos a rancidez oxidativa Draper et al. identificaron $\mathrm{N}-\varepsilon-(2$ propenal)-lisina como forma principal de malondialdehído, un producto de la oxidación lipídica (27), en la digestión de los alimentos (28). Este compuesto se forma aparentemente por reacción entre el MDA generado en la rancidez oxidativa de los alimentos y los grupos $\varepsilon$-amino libres de las proteínas. Estudios de tránsito metabólico de este compuesto han revelado que el mismo se excreta en la orina parcialmente inmodificado (29) y parcialmente como su $\mathrm{N}$ - $\alpha$-acetil derivado (30).

Dado que las pérdidas de lisina son debidas a productos secundarios de oxidación lipídica, se puede pensar que el alimento se convertirá en inaceptable organolépticamente antes que el daño al valor nutritivo de la 
proteína haya disminuido en alguna extensión. Esto, sin embargo, puede no ser el caso de ciertos alimentos animales como son los pescados o los alimentos destinados a consumo animal donde las pérdidas de lisina podrian ser importantes. Por otra parte, estas reacciones de interacción pueden asimismo provocar una disminución en el olor característico de algunos de los productos secundarios de la oxidación lipídica (31).

\section{POSIBLE FORMACION DE PRODUCTOS TOXICOS}

En los últimos años se ha incrementado notablemente el interés en el estudio de las dietas ricas en ácidos grasos y su posible relación con ciertas condiciones patológicas, incluyendo enfermedades coronarias y el cáncer. No obstante, hay pocos estudios científicos que traten de los efectos toxicológicos y fisiológicos de los productos de oxidación de los lípidos $(32,33)$ y menos aún de los productos de interacción entre lípidos oxidados y proteínas.

Ciertas proteínas pueden actuar como catalizadores en la oxidación lipídica por lo que, en cierta manera, pueden acelerar aquel proceso. Los hidroperóxidos lipídicos son moderadamente tóxicos cuando son administrados intravenosamente (34) siendo el ácido linoleico autooxidado el más tóxico de los mismos (32). La toxicidad de ellos parece que es paralela a la facilidad con que son reducidos. Así, por ejemplo, el peróxido de t-butilo, que sólo es reducido con considerable dificultad, es muy poco tóxico. Esto puede ser debido a que la reducción de estos materiales (por ejemplo, con iones ferrosos o mediante el sistema citocromo $\mathrm{P}-450$ ) produciría radicales alcoxi que son mucho más reactivos y menos selectivos que los peroxilos (35).

Durante mucho tiempo ha existido controversia sobre la capacidad de los hidroperóxidos de atravesar la barrera intestinal. Sin embargo, recientemente Nakatsugawa y Kaneda (36) encontraron que el 0,23\% del hidroperóxido del linoleato de metilo administrado a conejos aparecía intacto en la linfa. Esto indica que una parte de los hidroperóxidos son absorbidos de la pared intestinal. En ratas, el hidroperóxido administrado fue un parte absorbido de la pared intestinal y en parte convertido en hidroxi- $y$ oxooctadecadienoatos de metilo que también fueron absorbidos (37).

Los productos secundarios de la oxidación lipídica de bajo peso molecular son absorbidos con mayor rapidez a causa de la menor longitud de la cadena carbonada que los hace más hidrofílicos que el correspondiente hidroperóxido (38). Entre estos productos secundarios de la oxidación lipídica, el malondialhdehído ha sido indudablemente el más estudiado debido a su reactividad con los grupos amino y sulfhidrilo de las proteínas, las bases púricas y pirimidínicas de los ácidos nucléicos y grupos nitrogenados de los fosfolípidos (39). El malondialdehído se encuentra en los alimentos en un rango de $<0.1$ a aproximadamente $10 \mathrm{ppm}$, dependiendo de su composición en ácidos grasos y de las condiciones de almacenamiento y procesamiento (40) (41), donde se forma en la oxidación de los ácidos grasos poliinsaturados. Este compuesto ha sido encontrado tóxico (39) y mutagénico (42) y ha sido relacionado con la formación de componentes fluorescentes del tipo lipofuscinas que se acumulan en los organismos vivos con la edad (43). No obstante, su potencial toxicidad ha sido recientemente discutida por Draper et al. que han encontrado que menos del $10 \%$ del malondialdehído formado en los alimentos está realmente en su forma libre (28). Como se citó anteriomente, la mayor parte se encuentra formando un compuesto con el grupo $\varepsilon$-amino de la lisina ( $\mathrm{N}-\varepsilon$-[2-propenal]-lisina) que es absorbido como tal y excretado parcialmente sin modificar y parcialmente $\mathrm{N}-\alpha$-acetilado. En este sentido, la formación de compuestos de interacción lípido-proteína puede mitigar la toxicidad de los lípidos oxidados aún a costa de perder valor nutricional.

Otro ejemplo en este sentido es la formación del ácido tiazolidín-4-carboxílico (TCA), también llamado tioprolina, por condensación de cisteína y formaldehído (44) (45). Este aminoácido ha sido encontrado en hígado de rata homogenizado incubado con cisteína (46) y ha sido considerado una forma de eliminar formaldehído (47). El TCA tiene efectos protectores del hígado en ratas contra agentes hepatotóxidos y efectos protectores del envejecimiento en Drosophila y ratón (48) (49). Es asimismo capaz de nitrosar con facilidad (varios cientos de veces más rápido que la prolina (50)) produciendo ácidos $\mathrm{N}$-nitrosotiazolidin-4-carboxílico (NTCA). Tanto el NTCA como su 2-metil derivado son compuestos que se encuentran habitualmente en la orina humana y no son mutagénicos (51). En la actualidad el TCA es considerado un eficaz agente captor de moléculas de nitrito en el cuerpo humano evitando la formación de los carcinogenéticos compuestos $\mathrm{N}$-nitrosados (52).

\section{AGRADECIMIENTOS}

Al Prof. Eduardo Vioque por sus valiosas discusiones durante la realización de este trabajo.

\section{BIBLIOGRAFIA}

1. Hidalgo, F.J; Zamora, R. y Alaiz, M.- "Modificaciones producidas en las proteinas alimenticias por su interacción con lípidos peroxidados. I. Química radicalaria de los ácidos grasos poliinsaturados".- Grasas y Aceites 42 (1991) 379-386.

2. Hidalgo, F.J., Zamora, R. y Alaiz, M.- "Modificaciones producidas en las proteínas alimenticias por su interacción con lipidos peroxidados. II. Mecanismos conocidos de la interacción lípido (oxidado)-proteína". Grasas y Aceites 43 (1992) 31-38.

3. Hurrel, R.F. y Finot, P.A.- "Effects of food processing on protein digestibility and amino acid availability" en "Digestibility and amino acid availability in cerals and oilseeds". pp. 233-246.- Finley, J.W. y Hopkins, D.T. (Eds), American Association of Cereal Chemists, Inc., St Paul, Minnesota, 1985.

4. Finley, J.W., Wheeler, E.L. y Witt, S.C.- "Oxidation of gluthatione by hydrogen peroxide and other oxidizing agents".- J. Agric. Food Chem. 29 (1981) 404-407.

5. Finley, J.W. y Ludin, R.E.- "Lipid hydroperoxides induced oxidation of cysteine in protein" en "Autoxidation in food and biological systems", pp. 223-235.- Simic, M.G. y Karel, M. (Eds), Plenum Press, New York, 1980.

6. Bennett, M.A.- "Metabolism of sulphur. VII. A quantitative study of the replaceability of L-cysteine by various sulphur containing amino acids in the diets of albino rats".- Biochem. J. 33 (1939) 885-892. 
7. Bennett, M.A.- "Metabolism of sulphur. V. The replaceability of L-cysteine in the diets of rats with some partially oxidized derivatives".- Biochem. J. 31 (1937) 962-965.

8. Miller, D.S. y Samuel, P.D.- "Effects of the addition of sulphur compounds to the diet on utilization of protein in young growing rats".- $J$ Sci. Food Agric. 21 (1970) 616-618.

9. Nielsen, H.K.; Loliger, J. y Hurrell, R.F.- "Reactions of proteins with oxidizing lipids. 1. Analytical measurements of lipid oxidation and of amino acid losses in a whey-methyl linoleate model system".- $\mathrm{Br} . \mathrm{J}$ Nutr. 53 (1985) 61-73.

10. Nielsen, H.K.; Finot, P.A. y Hurrell, R.F.- "Reactions of proteins with oxidizing lipids. 2. Influence on protein quality and on the bioavailability of lysine, methionine, cyst(e)ine and tryptophan as measured in rat assays".- Br. J. Nutr. 53 (1985) 75-86.

11. Finot, P.A.- "Nutritional and metabolic aspects of protein modification during food processing" en "Modificaton of proteins. Food, nutritional and pharmacological aspects", pp. 91-124.- Feeney, R.E. y Whitaker J.R. (Eds), American Chemical Soc., Washington D.C., 1982

12. Bennett, M.A.- "The replaceability of dl-methionine in the diet of the albino rat with dl-methionine sulfone and dl-methionine methylsulfonium chloride".- J. Biol. Chem. 141 (1941) 573-578.

13. Bennett, M.A.- "Metabolism of sulfur. X. The replaceability of dl-methionine in the diet of albino rats with the partially oxidized derivative, dl-methionine sulfoxide".- Biochem. J. 33 (1939) 1794-1797.

14. Miller, S.A., Tannenbaum, S.R. y Seitz, A.W.- "Utilization of L-methionine sulfoxide by the rat".- J. Nutr. 100 (1970) 909-916.

15. Gjoen, A.U. y Njaa, L.R.- "Methionine sulfoxide as a source of sulfur-containing amino acids for the young rat".- Br. J. Nutr. 37 (1977) 93-105.

16. Aymard, C.; Seyer, L. y Cheftel, J.C.- "Enzymic reduction of methionine sulfoxide. In vitro experiments with rat liver and kidney".- Agric Biol. Chem. 43 (1979) 1869-1872.

17. Cuq, J.L.; Besancon, P.; Chartier, L. y Cheftel, J.C.- "Oxidation of methionine residues of food proteins and nutritional availability of protein-bound methionine sulfoxide".- Food Chem. 3 (1978) 85-102.

18. Ronzio, R.A.; Rowe, W.B. y Meister, A.- "Mechanism of inhibition of glutamine synthetase by methionine sulfoximine".- Biochemistry 8 (1969) 1066-1075

19. Richman, P.G.; Orlowski, M.; Meister, A.- "Inhibition of $\gamma$-glutamilcysteine synthetase by L-methionine-S-sulfoximine".- J. Biol. Chem. 248 (1973) 6684-6690.

20. Food and Agriculture Organization.- "Amino acid content of foods and biological data on proteins".- FAO, Rome, 1970.

21. O'Brien, P.J.- "The effects of hydrogen peroxide or lipid peroxide on cytochrome C".- Biochem. J. 101 (1966) 12P-13P

22. Kanazawa, K.; Danno, G. y Natake, M.- "Lysozyme damage caused by secondary degradation products during the autoxidation process of linoleic acid". - J. Nutr. Sci. Vitaminol. 21 (1975) 373-382.

23. Nielsen, H.K.; De Weck, D.; Finot, P.A.; Liardon, R. y Hurrell, R.F."Stability of tryptophan during food processing and storage. 1. Comparative losses of tryptophan, lysine and methionine in different mode systems".- Br. J. Nutr. 53 (1985) 281-292.

24. Nielsen, H.K.; Klein, A. y Hurrell, R. F.- "Stability of tryptophan during food processing and storage. 2. A comparison of methods used for the measurement of tryptophan losses in processed foods".- $\mathrm{Br} . \mathrm{J}$. Nutr. 53 (1985) 293-300

25. Horigome, T. y Miura, M.- "Interaction of protein and oxidized ethy linoleate in the dry state and the nutritive value of the reacted protein". J. Agric. Chem. Soc. Japan 48 (1974) 437-444.

26. Chiba, H.; Doy, H.; Yoshikawa, M. y Sugimoto, E.- "Deterioration of casein components by malonaldehyde".- Agric. Biol. Chem. 40 (1976) $1001-1010$.

27. Gómez-Sánchez, A.: Hermosín, I, y Maya, I.- "Cleavage and oligomerization of malondialdehyde under physiological conditions". - Tetrahedron Lett. 31 (1990) 4077-4080.

28. Piché, L.A.; Cole, P.D.; Hadley, M.; Van den Bergh, R. y Draper $\mathrm{H} . \mathrm{H}$.- "Identification of $\mathrm{N}-\mathrm{\varepsilon}-$ (2-propenal) lysine as the main form of malondialdehyde in food digesta".- Carcinogenesis 9 (1988) 473-477.
29. Draper, H.H: Hadley, M. Lissemore L: Laing, N.M y Cole, P.D. "Identification of $\mathrm{N}-\varepsilon$-(2-propenal) lysine as a major urinary metabolite of malondialdehyde". - Lipids 23 (1988) 626-628.

30. McGirr, L.G.; Hadley, M. y Draper, H.H.- "Identification of $\mathrm{N}-\alpha$-acetyl- $\varepsilon$-(2-propenal)lysine as an urinary metabolite of malondialdehyde".- J. Biol. Chem. 260 (1985) 15427-15431.

31. Pokorny, J. y Davidek, J.- "Influence of interactions of proteins with oxidized lipids on nutrition and sensory value of foods".- Acta Alimentaria Polonica 5 (1979) 87-95.

32. Pryor, W.A.- "The toxicity of oxidized fats: lipid hydroperoxides, their decomposition products and their co-oxidation products" en "Proccedings of the XIII International Congress of Nutrition", pp. 589-593. Taylor, T.G. y Jenkins, N.K. (Eds), John Libbey and Company, Ltd. London, 1986.

33. Kubow, S.- "Toxicity of dietary lipid peroxidation products".- Trends Food Sci. Technol. 1 (1990) 67-71.

34. Cortesi, R. y Privett, O.S.- "Toxicity of fatty ozonides and peroxides". Lipids 7 (1972) 715-721

35. Pryor, W.A.- "Free radicals in autoxidation and in aging" en "Free radicals in molecular biology, aging and disease".- Armstrong, D. Sohol, R.S.; Cutler, R.G.; Slater, T.F. (Eds), Raven Press, New York 1984.

36. Nakatsugawa, K. y Kaneda, T.- "Absorption of methyl linoleate hydroperoxides in rabbit".- Yukagaku 30 (1981) 74-77.

37. Nakatsugawa, K. y Kaneda, T.- "Absorption and metabolism of methy linoleate hydroperoxides in rats".- Yukagaku 32 (1983) 361-366.

38. Oarada, M.; Miyazawa, T. y Kaneda, T.- "Distribution of ${ }^{14} \mathrm{C}$ after oral adminstration of $\left(\mathrm{U}-{ }^{14} \mathrm{C}\right)$ labeled methyl linoleate hydroperoxides and their secondary oxidation products in rats".- Lipids 21 (1986) 150-154.

39. Draper, H.H. McGirr, L.G. y Hadley, H.- "The metabolism of malondialdehyde".- Lipids 21 (1986) 305-307.

40. Shamberger, R.J.; Shamberger, B.A. y Willis, C.E.- "Malonaldehyde content of food".- J. Nutr. 107 (1977) 1404-1409.

41. Siu, G.M. y Draper, H.H.- "A survey of the malonaldehyde content of retail meats and fish".- J. Food Sci. 43 (1978) 1147-1149.

42. Basu, A.K. y Marnett, L.J.- "Unequivocal demostration that malondialdehyde is a mutagen".- Carcinogenesis 4 (1983) 331-333.

43. Tappel, A.L.- "Lipid peroxidation damage to cell components".- Fed. Proc. 32 (1973) 1870-1874.

44. Schubert, M.P.- "Compounds of thiol acids with aldehydes".- J. Biol. Chem. 114 (1936) 341-350.

45. Ratner, S.; Clarke, H.T.- "The action of formaldehyde upon cysteine".- J. Am. Chem. Soc. 59 (1937) 200-209.

46. Caballini, D.; DeMarco, C.; Mondovi, B. y Trasarti, F.- "Studies of the metabolism of thiazolidine carboxylic acid by rat liver homogenate". Biochim. Biophys. Acta 22 (1956) 558-564.

47. Debey, H.J.; Mackenzie, J.B. y Mackenzie, C.G.- "The replacemen by thiazolidinecarboxylic acid of exogenous cystine and cysteine".- $J$. Nutr. 66 (1958) 607-619.

48. Siegers, C.P.; Strubelt, O. y Völpel, M.- "The antihepatotoxic activity of dithiocarb as compared with six other this compounds in mice". Arch Toxicol. 41 (1978) 79-88.

49. Miquel, J.; Fleming, J. y Economos, A.C.- "Antioxidants, metabolic rate and aging in Drosophila".- Arch. Gerontol. Geriatr. 1 (1982) 159-165.

50. Ohshima, H: O'Neil, I.K. Friesen, M.; Bérèziat, J.C. y Bartsch, H. "Occurrence in human urine of new sulphur-containing $\mathrm{N}$-nitrosamino acid and its 2-methyl derivative, and their formation".- J. Cancer Res. Clin. Oncol. 108 (1984) 121-128.

51. Tahira, T.; Tsuda, M.; Wakabayashi, K.; Nagao, M. y Sugimura, T. "Kinetics of nitrosation of thioproline; the precursor of a major nitroso compound in human urine and its role as a nitrite scavenger".- Gann 75 (1984) 889-894.

52. Kurashima, Y.; Tsuda, M. y Sugimura, T.- "Marked formation of thiazolidine-4-carboxylic acid, an effective nitrite trapping agent in vivo on boiling of dried Shiitake mushroomn".- J. Agric. Food Chem. 38 (1990) 1945-1949.

(Recibido: Diciembre 1990) 